\title{
FIELD INITIATION DESIGN FUNDAMENTALS FOR PULSED ALTERNATORS
}

By:

J.R. Kitzmiller

M.D. Driga

13th Electromagnetic Launch Technology Symposium, Potsdam (Berlin), Germany, May 22-25, 2006

IEEE Transactions on Magnetics, vol. 43, no. 1, January 2007, pp. 246-249

PR 413

Center for Electromechanics

The University of Texas at Austin

PRC, Mail Code R7000

Austin, TX 78712

(512) 471-4496 


\title{
Field Initiation Design Fundamentals for Pulsed Alternators
}

\author{
Jon R. Kitzmiller and Mircea D. Driga
}

\begin{abstract}
Efficient high performance pulsed alternator (PA) systems have low impedance field windings that rely on very fast current rise times in order to maintain attractive system efficiencies. These systems rely on positive feed back self-excitation, or 'bootstrapping' action, to energize the field winding. The self-excitation process is typically started by a small capacitor based power supply which is discharged (or seeded) directly into the field winding. The design of this power supply, often called the Field Initiation Module (FIM) is critically important to the process of self-excitation.

Augmented by numerical simulations, this paper examines the important aspects to consider when designing a proper FIM including:

- Impact on system efficiency

- Minimum rotor speed for proper FIM function

- Control schemes for triggering the FIM

- Proper operating voltage for the FIM
\end{abstract}

\section{INTRODUCTION}

Air-core pulsed alternators (PAs), with or without compensation, rely on selfexcited, very high MMF field windings to provide excitation. As PA designs mature toward higher energy and power densities, the resulting power required to charge the field windings efficiently has also increased. Field charging power levels exceeding 300 MW have been demonstrated [1] in the laboratory, and future designs far exceeding this level of charging power are expected. An external dc power source is not practical, so PAs must rely on the self-excitation process to energize the field windings.

The role of the FIM then becomes apparent. It energizes the field winding with some minimal level of current in order to initiate the self-excitation process. The FIM is typically a small, self-contained, capacitor-based power supply that is tied directly into the field winding. When discharged, the current in the field winding rises, thus inducing armature voltage. The discharge controller then begins to command the field coil converter switches in order to rectify armature currents directly back into the field 
winding. This is a positive feedback process and must be carefully controlled. This paper investigates the design requirements of the FIM for efficient PA operation.

\section{FIM MODEL}

A railgun performance simulation based upon the use of PAs was constructed within the MATLAB/Simulink ${ }^{\circledR}$ environment. The specific design of the PA used is tactically uninteresting and based upon previous studies [2], [3], [4] conducted at the Center for Electromechanics of The University of Texas at Austin (UT-CEM). The model block representing the FIM of a typical PA is shown in figure 1. Because version 6 of MATLAB does not permit modeling a pre-charged capacitor, the capacitor model is charged dynamically at the beginning of the simulation. In practice, the FIM capacitor is typically pre-charged for the first shot (before or during rotor motoring) and charged dynamically by the field winding for burst operation.

Referring to the circuit shown in figure 1, there is a current-limiting resistor $(0.14 \Omega)$ in series with a $7 \mathrm{kV}$ voltage source. The value of the dc voltage source was varied to provide in this case $4.5 \mathrm{kV}$ across the terminals of the field winding at FIM initiation (covered in detail in a later section of this paper). An ideal SCR switch was gated at the start of the simulation to charge the capacitor. Also, there will typically be a series impedance between the seed capacitor and the field winding. As discussed in this paper, the design of this impedance can be tailored specifically to best suit the requirements of the system.

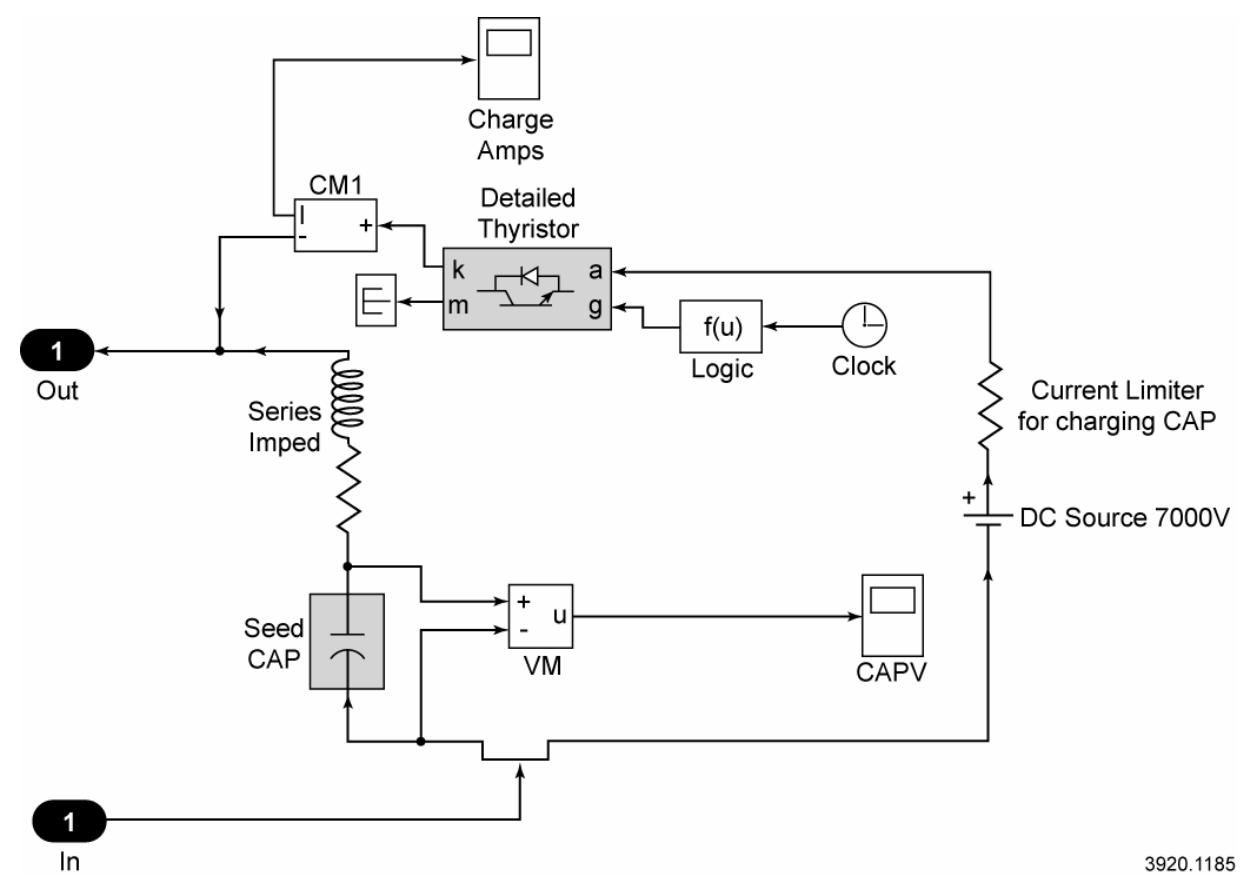

Figure 1. FIM block as it resides within Simulink ${ }^{\circledR}$ 


\section{IMPACT ON SYSTEM EFFICIENCY}

In order to expedite the study, a first order approximation for the charging current profile was utilized. The field winding self-excitation process follows the exponential profile approximated by [5];

$$
i(t)=I_{0} e^{\alpha t}
$$

where

$$
\begin{aligned}
I_{0} & =\text { initial dc seed current starting point } \\
t & =\text { time } \\
\alpha & =\text { charging coefficient }
\end{aligned}
$$

The charging coefficient is a constant that depends on the magnetic coupling between the field and armature windings, the dc field impedance values, and rotor speed. The calculated value for the charging coefficient for the study PA is plotted in figure 2. Changing the calculated values to those produced in simulation resulted in the estimated current profile shown in figure 3. The seed current value is $15 \mathrm{kA}$. This curve was compared to results from the Simulink simulation and proved a close match to those results. Finally, the ohmic losses from this profile were obtained by squaring and integrating the current profile times the field resistance. These results are shown in figure 4.

The calculated charging losses detailed above are roughly $300 \mathrm{~kJ}$ for the study PA. Simulated results indicated a value within $0.5 \%$ of the calculated losses. Meanwhile, the simulation predicted a total of $1.13 \mathrm{MJ}$ of total field energy losses, so that the charging cycle accounts for about $25 \%$ of the total field losses.

The seed current was reduced to $5 \mathrm{kA}$, and figures 5 and 6 show the results. Although t he charging time increased from $16 \mathrm{~ms}$ to $23 \mathrm{~ms}$, the losses remained about the same. This is borne out by the strong exponential in the cycle. As figure 7 shows, at $1 \mathrm{kA}$ seed current, the losses are still about $300 \mathrm{~kJ}$. What is happening is that the time to achieve the desired field is very much extended. Ultimately, this could result in an undesirable feel for the gunner, and an undesirable increase in brush drag, heating, and wear. The results from this are clear: the field efficiency is not influenced strongly by the seed current. 


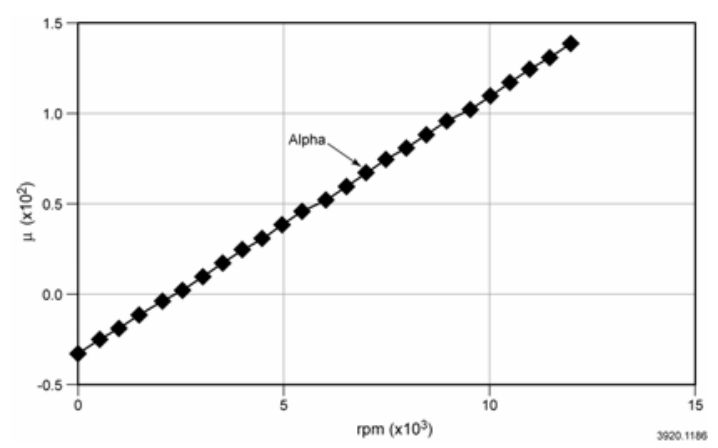

Figure 2. Charging coefficient vs. rpm for the study PA

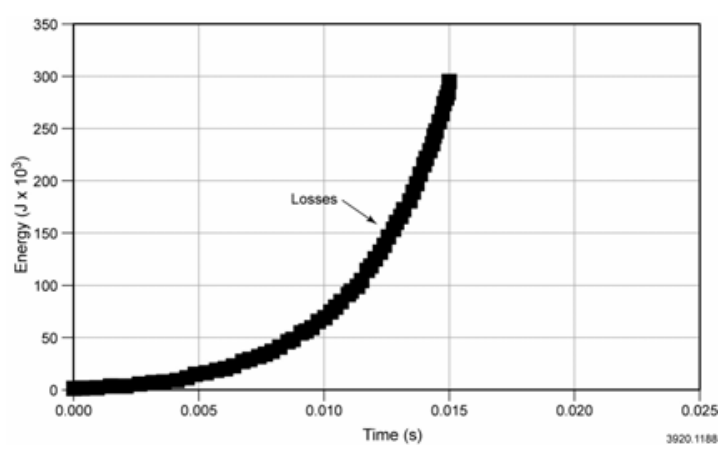

Figure 4. Calculated charging losses vs. time for the study PA field coil

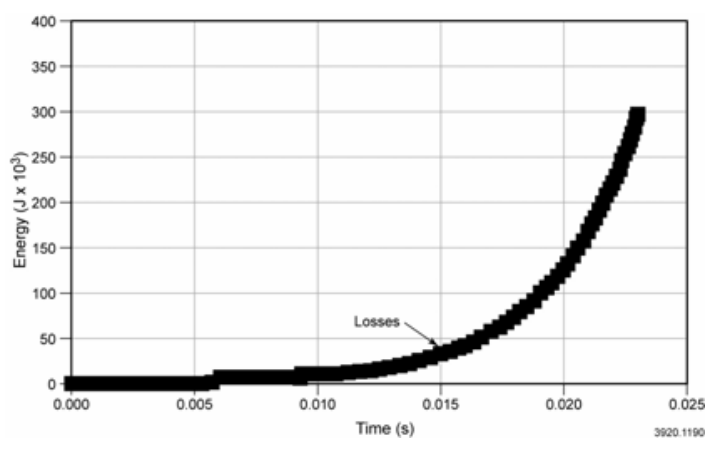

Figure 6. Charging losses vs. time for $5 \mathrm{kA}$ seed current

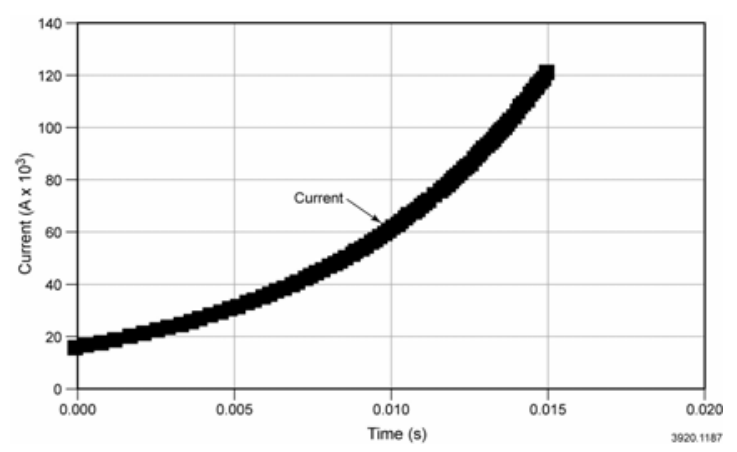

Figure 3. Charging current profile vs. time estimation

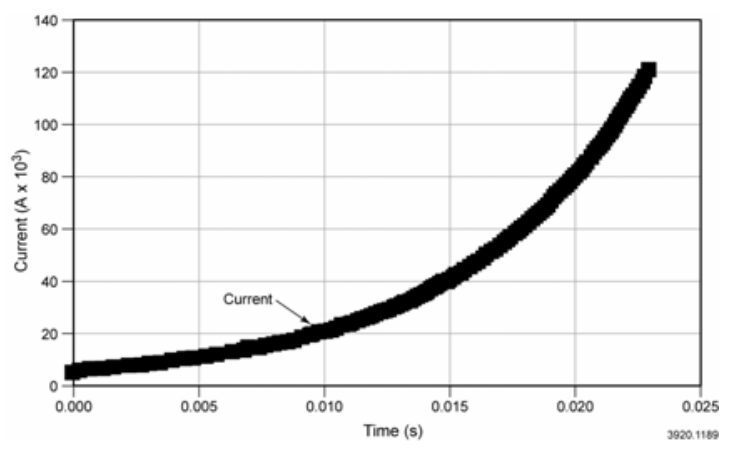

Figure 5. Charging current profile vs. time for 5 kA seed current

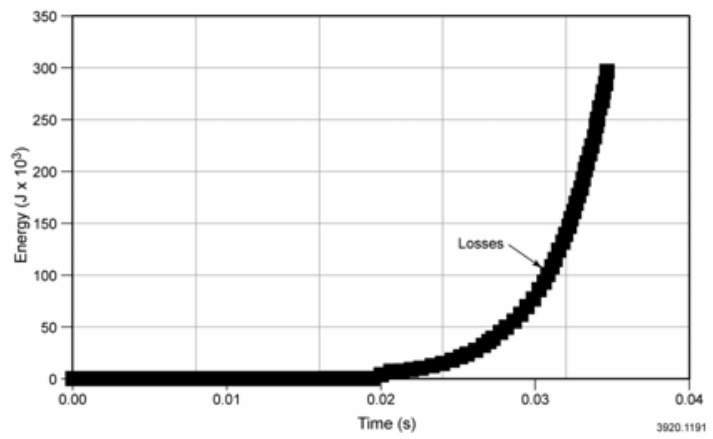

Figure 7. Charging losses vs. time for $1 \mathrm{kA}$ seed current 


\section{SEED CURRENT AND Rotor SPEED (VOLTAGE) ISSUES}

Rotor speed is another important aspect in selecting the proper seed current level. Once again the main purpose of the FIM is to provide a seed current to the field winding so that the PA can begin the self-excitation process. And as discussed above, it is a strong function of rotor speed. In general, the PA can provide power to launch other lower-velocity rounds from diminished speeds. In addition, for subsystem commissioning and maintenance mode purposes, UT-CEM engineers routinely operate the PA at a much reduced speed. Based upon these factors, a minimum operating speed of approximately $50 \%$ of the PA design full speed is recommended.

The next issue is the minimum voltage required from the PA to initiate excitation. This depends somewhat on the bus line impedances and field coil converter (FCC) characteristics. Again, experience with these systems has led UT-CEM engineers to conclude that there should be at least $25 \mathrm{~V}$ across each thyristor in the FCC to commence the self-excitation. Voltages less than $25 \mathrm{~V}$, when combined with particular gate impulses, have resulted in faulty turn-on of SCRs during testing.

According to figure 2, at 6,000 rpm the study PA still has a strong positive charging coefficient of 54. To study the effectiveness of this, a $200 \mu \mathrm{F}$ capacitor charged to $5 \mathrm{kV}$ was used in the simulation. The resulting voltage profile and start of rectification is shown in figures 8 and 9. Note that the rectification was timed to occur very near the peak seed current location; otherwise, this should be a valid seed current design point.

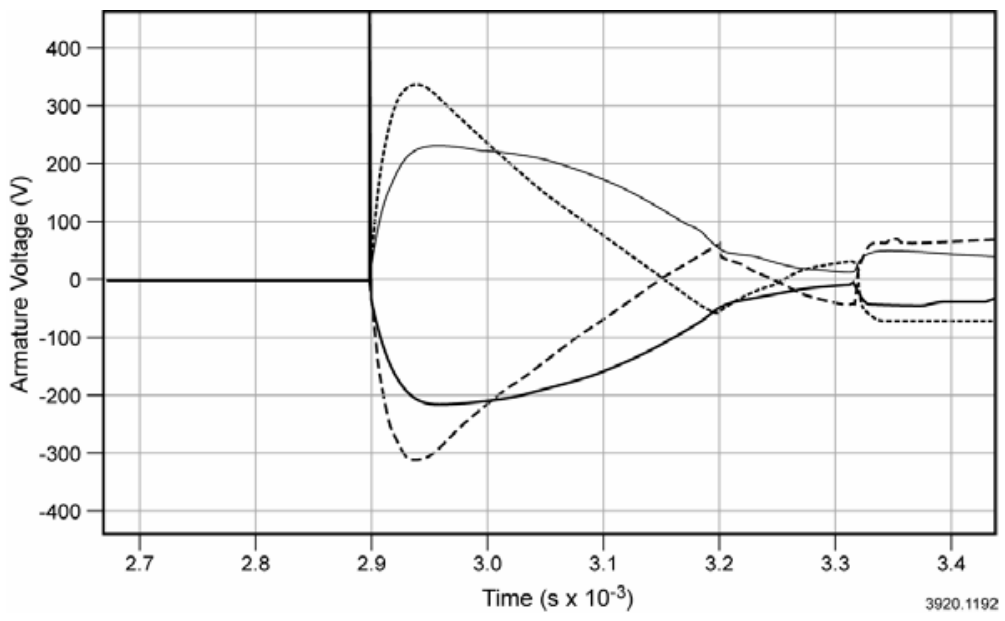

Figure 8. Voltage vs. time from $200 \mu \mathrm{F}$ cap @ 5kV discharging into study PA at 6,000 rpm 


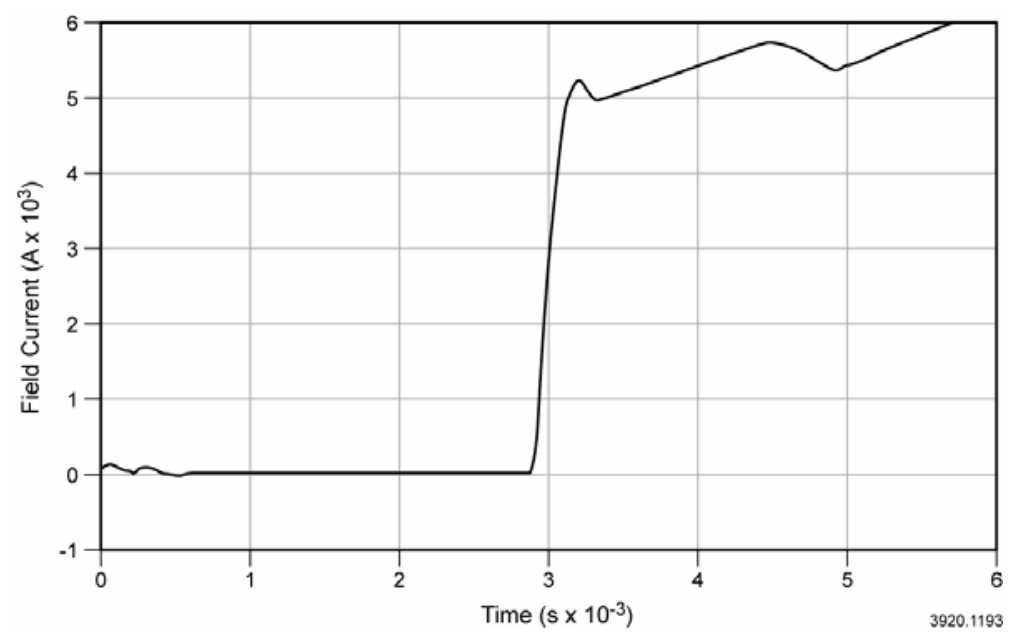

Figure 9. Field current vs. time from $299 \mu$ F FIM event of figure 8

\section{SELF EXCITATION AND CONTROL SCHEME}

In the simulation results, the FIM discharge was set so that the PA had a switch trigger that occurred near the peak of the seed current cycle. This meant that for such a low seed current impulse, the controller would have to consider the speed of the machine and the time of the FIM discharge event, so that a phase trigger signal coincided at or just after the peak of the seed current profile. While this is certainly possible, it does add a level of complexity into the controller design and software.

In previous PA systems, the start of the self-excitation process was passive, i.e., the seed current pulse was of long enough duration so that a phase trigger always occurred at or just after peak current was observed. At 6,000 rpm, a phase is available to be triggered every $1.25 \mathrm{~ms}$. For reliable triggering, a random phase of the seed current impulse must last for more that $2.5 \mathrm{~ms}$. For the simulation that produced figures 10 and 11 , the capacitance was set at $2,500 \mu \mathrm{F}$ at $5 \mathrm{kV}$ charge. The phase 4 trigger sequence occurs just before peak current and phase 1 is then able to initiate the bootstrapping sequence at the $3 \mathrm{kA}$ field current level. As stated above, this value is at the bottom range of the desired starting voltage.

It should also be mentioned that the circuit parameters of the FIM itself can be adjusted to optimize the pulse width and current delivered to the field coil, in order to minimize the capacitance required. There are additional trade-offs to be considered if FIM adjustment is to be implemented; however, if a very low capacitance is required (approaching $10 \%$ of the value defined earlier), a control modification would definitely be warranted. 


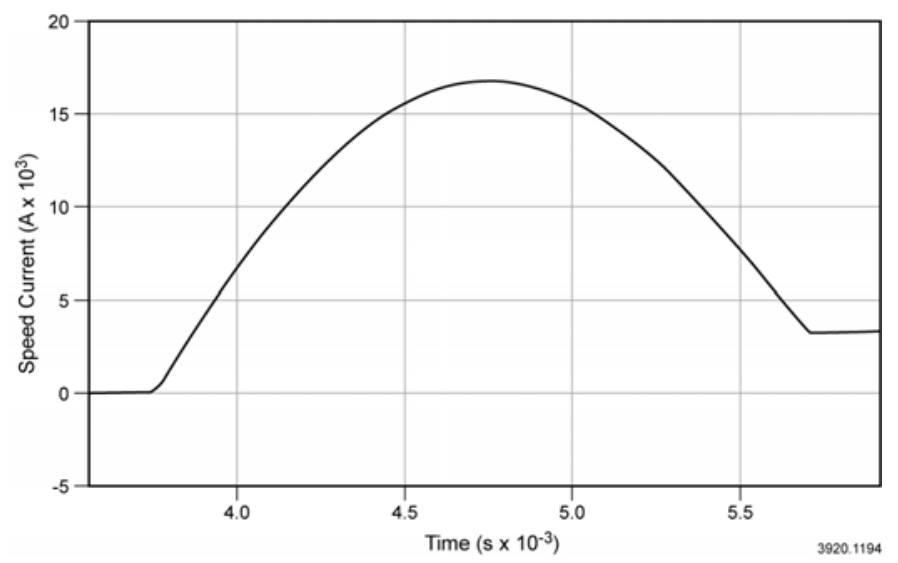

Figure 10. Seed current for $2,500 \mu \mathrm{F}$ and $5 \mathrm{kV}$

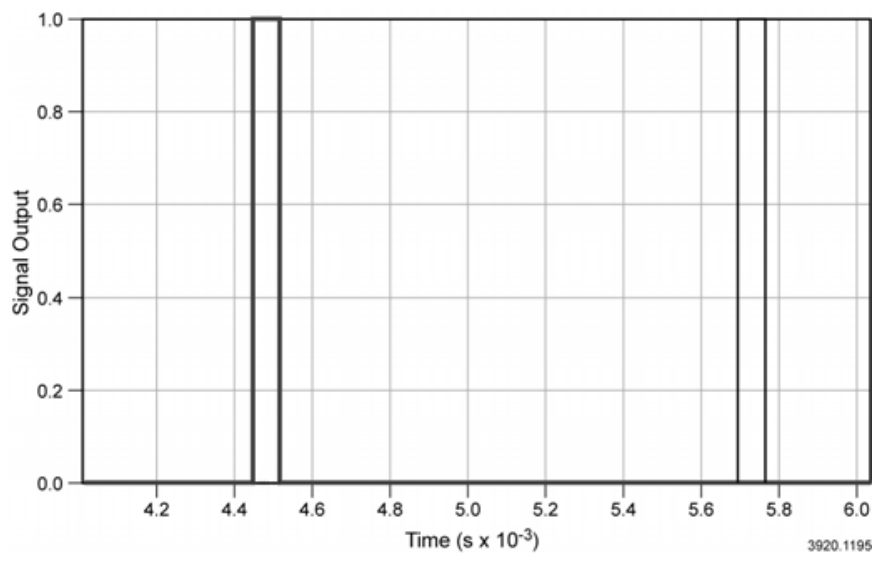

Figure 11. Phase 4 and 1 FCC trigger points

From the results of this simulation, it can be concluded that there is a strong dependence on the size of the FIM with the control scheme selected. Minimizing seed capacitance would seem to necessitate adapting a smart control algorithm to time the phase sequencing with the FIM discharge.

\section{FIM CHARGE VOLTAGE LEVELS}

UT-CEM engineers recommend a charge voltage commensurate with the maximum charge voltage of the field winding. Capacitor-stored energy is a strong function of voltage, but there is also an inherent operational fault protection advantage to be gained by using this voltage level.

When the properly designed FIM is discharged into the field winding, the capacitor voltage is seen instantly across the field winding. If there is a brush or insulation issue at this stage, the fault can be detected early and the discharge terminated with minimal risk to the generator and its subcomponents. The voltage seen across the field winding for the case of 2,500 $\mu \mathrm{F}$ FIM discharge is shown in figure 12 . 


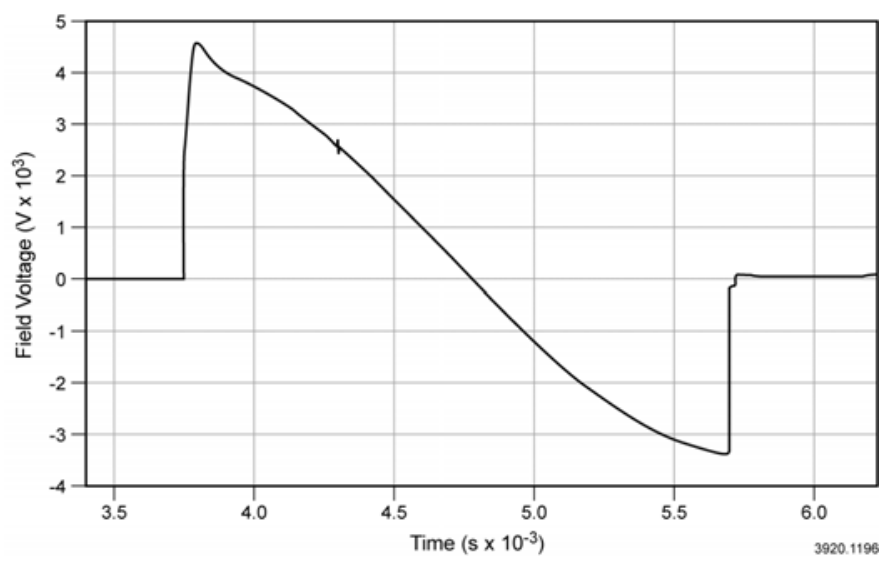

Figure 12. Field Volts during 2,500 $\mu \mathrm{F}$, 5kV FIM discharge

\section{SIMULATION VERIFICATION}

The $200 \mu \mathrm{F}$ FIM case was loaded into the performance simulation for the study $\mathrm{PA}$ to make sure the assumptions proved to be adequate. Figure 13 shows the difference between field charging characteristics in the two cases (2,500 and $200 \mu \mathrm{F})$. The results verify that lower seed current will result in longer charging durations for the field winding. Figure 14 compares the two rotor energies extracted during discharge and demonstrates that there is little net difference produced by using the lower seed current. In fact, the lower seed current case shows slightly less net energy used because the initial shot phasing was slightly different, based on different seed starting points. Time did not permit correcting this descrepancy for the purpose of the study, and it was felt that identifying the general trend was more important.

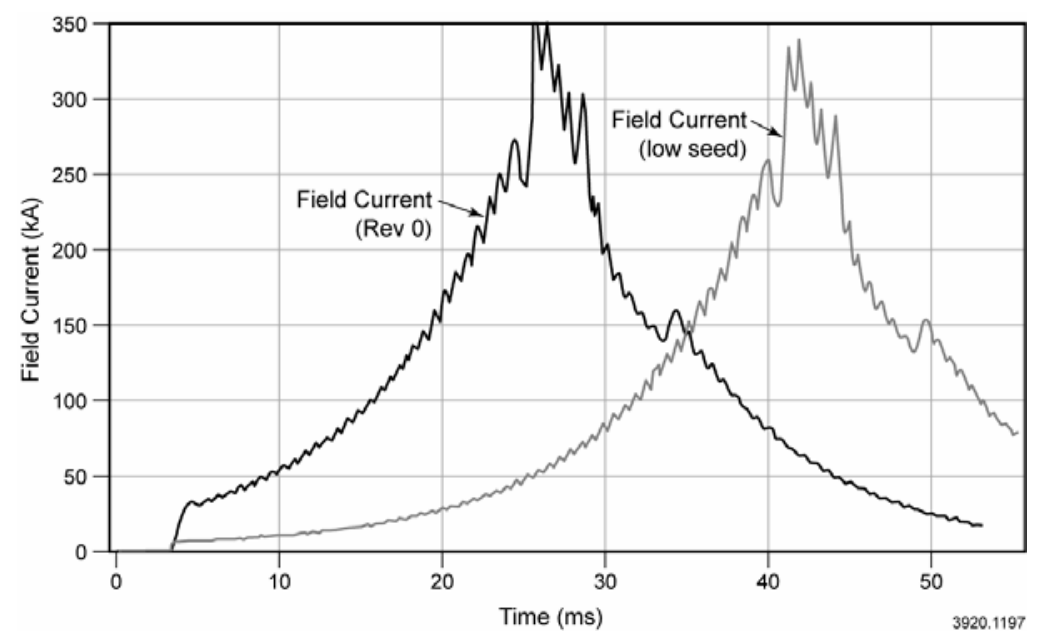

Figure 13. Current comparisons from simulation for $4,500 \mu \mathrm{F}$ and $200 \mu \mathrm{F}$ seed capacitors 


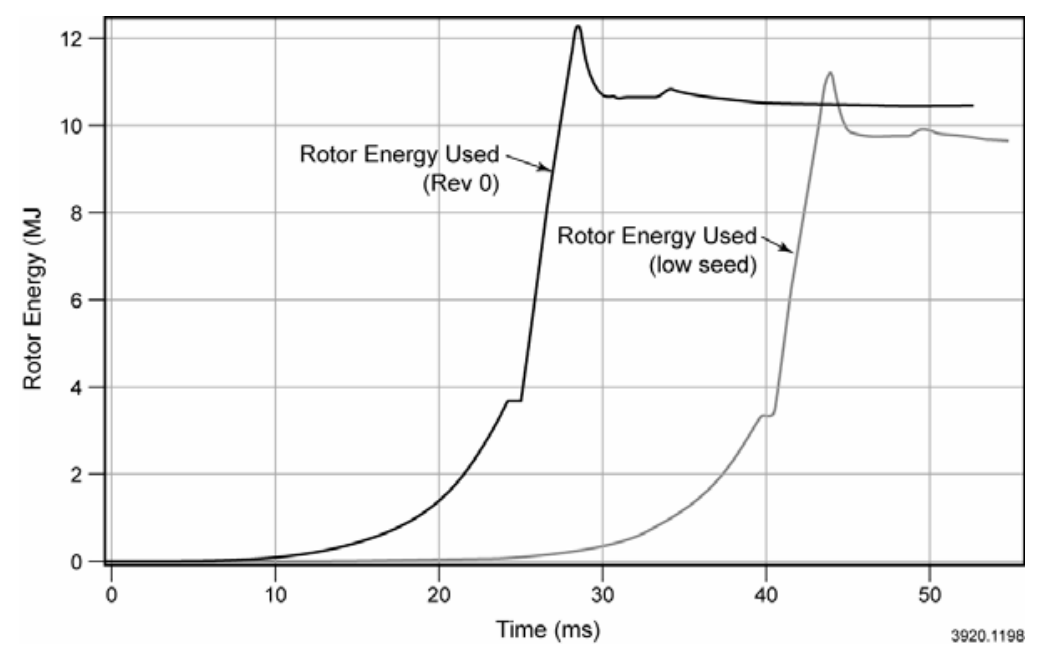

Figure 14. Rotor energy comparison for 4,500 $\mu \mathrm{F}$ and $200 \mu \mathrm{F}$ seed capacitors

\section{CONCLUSIONS AND RECOMMENDATIONS}

This paper addressed the general design guidelines of the FIM for any generic PA design. The guidelines can be summarized as following, and were verified through simulation:

1. The FIM should be designed so that the PAs can boot-strap from half of the rotor full design speed.

2. The FIM should be sized so that a minimum of $25 \mathrm{~V}$ appears across all SCRs located in the FCC.

3. The FIM seed capacitor size depends heavily on what control scheme is utilized; passive or calculated (controlled) timed FIM discharge.

Items 2 and 3 are interdependent and must be considered jointly for a successful design.

\section{ACKNOWLEDGEMENT}

This research was funded by the U.S. Army and managed by ARDEC, located at Picatinny Arsenal in New Jersey.

\section{REFERENCES}

[1] J.R. Kitzmiller, et al., "Predicted vs. actual performance of a model scale compulsator system," IEEE Transactions on Magnetics, vol. 37, no. 1, January 2001, pp. 362-366. 
[2] W.F. Weldon, et al., "Fundamental limitations and design considerations for compensated pulsed alternators," 2nd IEEE International Pulsed Power Conference, Lubbock, Texas, U.S.A., June 12-14, 1979.

[3] S.B. Pratap, et al., "Operating modes for compulsator based electromagnetic launcher systems," Digest of Technical Papers, 10th IEEE International Pulsed Power Conference, July 3-6, 1995, vol. 1, pp. 180-185.

[4] J.R. Kitzmiller, S.B. Pratap, and M.D. Driga, "An application guide for compulsators,” IEEE Transactions on Magnetics, vol. 39, no. 1, January 2003, pp. 285-288.

[5] S.B. Pratap, "Limitations on the minimum charging time for the field coil of air core compensated pulsed alternators," IEEE Transactions on Magnetics, vol. 27, no. 1, January 1991, pp. 365-368. 